\title{
Spin and pseudospin symmetries in the antinucleon spectrum of nuclei
}

\author{
R. Lisboa \\ Escola de Ciências e Tecnologia, Universidade Federal do Rio Grande do Norte, 59014-615 Natal, Rio Grande do Norte, Brazil and \\ Departamento de Física, Instituto Tecnológico de Aeronáutica, Centro Técnico Aeroespacial, \\ 12228-900 São José dos Campos, São Paulo, Brazil

\begin{abstract}
Departamento de Física, Instituto Tecnológico de Aeronáutica, Centro Técnico Aeroespacial, 12228-900 São José dos Campos, São Paulo, Brazil
\end{abstract} \\ M. Malheiro \\ P. Alberto and M. Fiolhais \\ Departamento de Física and Centro de Física Computacional, Universidade de Coimbra, P-3004-516 Coimbra, Portugal
}

A. S. de Castro

Departamento de Física e Química, Universidade Estadual Paulista, 12516-410 Guaratinguetá, São Paulo, Brazil (Received 10 February 2010; revised manuscript received 20 May 2010; published 28 June 2010)

\begin{abstract}
Spin and pseudospin symmetries in the spectra of nucleons and antinucleons are studied in a relativistic mean-field theory with scalar and vector Woods-Saxon potentials, in which the strength of the latter is allowed to change. We observe that, for nucleons and antinucleons, the spin symmetry is of perturbative nature and it is almost an exact symmetry in the physical region for antinucleons. The opposite situation is found in the pseudospin symmetry case, which is better realized for nucleons than for antinucleons, but is of dynamical nature and cannot be viewed in a perturbative way for either nucleons or antinucleons. This is shown by computation of the spin-orbit and pseudospin-orbit couplings for selected spin and pseudospin partners in both spectra.
\end{abstract}

DOI: 10.1103/PhysRevC.81.064324

PACS number(s): 21.10.Hw, 21.60.Cs, 03.65.Pm, 13.75.Cs

\section{INTRODUCTION}

Spin and pseudospin symmetries of the Dirac equation with scalar, $S$, and vector, $V$, potentials are observed, respectively, when the difference, $\Delta=V-S$, or the sum, $\Sigma=V+S$, is a constant. These constants are zero for bound systems whose potentials go to zero at infinity. Generally, in physical systems with this kind of potential neither of these conditions is met exactly but, in some cases, one of them can be approximately true. As Ginocchio pointed out, these symmetries may explain degeneracies in some heavy meson spectra (spin symmetry) or in single-particle energy levels in nuclei (pseudospin symmetry), when these physical systems are described by relativistic mean-field theories with scalar and vector potentials [1]. In terms of the nonrelativistic quantum numbers $n, l, j$ (i.e., the quantum numbers of the upper component of the Dirac spinor), exact spin symmetry means that the doublets $(n, l, j=l-1 / 2)(n, l, j=l+1 / 2)$ are degenerate (no spin-orbit coupling), while in the case of pseudospin symmetry the degeneracy refers to the doublets $\left(n^{\prime}, l+2, j=l-1 / 2\right)(n, l, j=l+1 / 2)$. In the latter case, a new principal quantum number $\tilde{n}$ and a new orbital angular momentum quantum number $\tilde{l}$ are defined, such that the doublets are labeled by $(\tilde{n}, \tilde{l}, \tilde{j}=\tilde{l} \pm 1 / 2)$. While $n, l$ are the common quantum numbers of the upper component of spin symmetry doublets, $\tilde{n}, \tilde{l}$ are the common quantum numbers of the lower component of the spinor of the pseudospin symmetry doublets. The relations among $n^{\prime}, \tilde{n}$, and $n$ may depend on the shape of the central mean field and the symmetry conditions, namely, whether it is a Woods-Saxon-like or a harmonic oscillator potential when there is pseudospin symmetry [2]. In the case of nuclei whose potentials are of the former type, those relations are given by $n^{\prime}=n-1, \tilde{n}=n-1$.

The study of the antinucleon spectrum in nuclei is of interest because of the conjectures about the existence of antinucleon bound levels inside the nucleus based on the relativistic description of nucleons. Naturally, this existence is controversial simply because of the annihilation that can occur between nucleons and antinucleons within nuclei [1]. Bürvenich et al. [3] have argued that, in a dense nuclear environment, the existence of bound states for antinucleons reduces the phase space available for such processes, and therefore bound antinucleons may live long enough to be observed experimentally. On the other hand, polarization measurements of the scattering of antiprotons on carbon [4] have indicated that the spin-orbit part of $N \bar{N}$ amplitudes is very small. As noted by Ginocchio [1], this may indicate that for the annihilation potential the vector part is similar to the scalar part, thus not destroying the high degree of spin symmetry that exists in antineutron spectra (see below). However, the true impact of the annihilation potential on the spectrum and even on the existence of antinucleon bound states in a nuclear mean field is not fully known.

With this in mind, in this paper we are going to examine in some detail the onset of spin and pseudospin symmetries in antinucleon single-particle states and compare it to what happens in the nucleon spectra. It has been shown [5-8] that pseudospin and spin symmetries are connected by charge conjugation since, under this operation, the sign of the vector 
potential $V$ is changed while the sign of the scalar potential $S$ remains the same, thus converting $\Delta$ into $-\Sigma$ and $\Sigma$ into $-\Delta$. This was shown explicitly for harmonic oscillator potentials [6]. Zhou et al. [5] have performed a realistic self-consistent mean-field calculation for antinucleons, showing how the shallow negative $\Sigma$ nucleon binding potential gives rise to a deep negative $-\Delta$ antinucleon binding potential, thereby holding many antinucleon single-particle states. One of the consequences of the magnitude of these potentials is that spin partners become almost degenerate, as was already noted by Ginocchio in his first review paper [8]. Recently, it was shown that spin symmetry is even higher for the anti- $\Lambda$ spectrum in a nucleus [9].

In this paper, we investigate charge-conjugation effects in spin and pseudospin symmetries in nuclei, in a unified way, by computing the energies of single-particle states of neutrons and antineutrons using scalar and vector Woods-Saxon potentials. The antineutron states are classified exactly in the same way as the neutron states, namely, by the quantum numbers of the corresponding spinor upper component, and the spin and pseudospin partners obtained accordingly. In this study we keep constant the range and diffusivity parameters that fit the neutron spectrum of ${ }^{208} \mathrm{~Pb}$, used in previous works [10,11], as well as the depth of the scalar potential, but vary the height of the vector potential. From this systematic study, we can assess the nature of the spin and pseudospin symmetries for nucleons and antinucleons, namely, its perturbative or nonperturbative nature. This is done by computing the contributions of the spin-orbit and pseudospin-orbit couplings to the energy splittings of spin and pseudospin partners, for both neutrons and antineutrons. A comparison between the radial functions of those partners is also made.

This paper is organized as follows. In Sec. II we present the general features of charge- conjugated solutions of the Dirac equation with spherical scalar and vector potentials and discuss their quantum numbers and their relation to pseudospin quantum numbers. In the subsequent section we present the numerical solutions of the Dirac equation for both particles and antiparticles in Woods-Saxon mean-field scalar and vector potentials with parameters that best fit the single-particle states of ${ }^{208} \mathrm{~Pb}$, but allowing for a variable strength of the vector potential. Finally, in Sec. IV, we draw the conclusions.

\section{CHARGE CONJUGATION IN THE DIRAC HAMILTONIAN WITH SCALAR AND VECTOR RADIAL POTENTIALS}

The Dirac Hamiltonian for a particle with mass $m$ under the action of external scalar, $S$, and vector, $V$, potentials reads $(\hbar=c=1)$

$$
H=\vec{\alpha} \cdot \vec{p}+\beta(m+S)+V,
$$

where $\vec{\alpha}$ and $\beta$ are the Dirac matrices. The time-independent Dirac equation for fermions, with energy $E$, is

$$
H \psi=E \psi .
$$

The charge-conjugation operator is given by $C=i \gamma^{2} K$, where $K$ is the complex conjugation operator [12]. When $C$ is applied to both sides of Eq. (2), one obtains the conjugate Dirac equation

$$
H_{\mathrm{c}} \psi_{\mathrm{c}}=-E_{\mathrm{c}} \psi_{\mathrm{c}},
$$

where the conjugated spinor is given by $\psi_{\mathrm{c}}=i \gamma^{2} \psi^{*}$ and the conjugate Hamiltonian is

$$
H_{\mathrm{c}}=\vec{\alpha} \cdot \vec{p}+\beta(m+S)-V,
$$

and $E_{\mathrm{c}}$ is the total energy related to the charge-conjugated spinor. From Eqs. (1) and (4), one concludes that the chargeconjugation operation changes the sign of the vector potential while keeping the sign of the scalar potential [6]. Therefore, the Hamiltonian is invariant under charge conjugation when there is only a scalar potential and, in such a case, the antifermion energies are symmetric with the corresponding fermion energies.

For spherically symmetric systems, the fermion Dirac spinor can be written as

$$
\begin{aligned}
\psi & =\left(\begin{array}{c}
i G_{n \kappa}(r) \phi_{\kappa m_{j}}(\theta, \varphi) \\
F_{n \kappa}(r) \vec{\sigma} \cdot \hat{r} \phi_{\kappa m_{j}}(\theta, \varphi)
\end{array}\right) \\
& =\left(\begin{array}{c}
i G_{n \kappa}(r) \phi_{\kappa m_{j}}(\theta, \varphi) \\
-F_{n \kappa}(r) \phi_{-\kappa m_{j}}(\theta, \varphi)
\end{array}\right),
\end{aligned}
$$

where $\phi_{\kappa m_{j}}(\theta, \varphi)$ are the spinor spherical harmonics and $G_{n \kappa}(r)$ and $F_{n \kappa}(r)$ are the radial wave functions for the upper and lower components. The $\kappa$ quantum number is related to the total angular momentum $j$ and orbital angular momentum $l$ through

$$
\kappa= \begin{cases}-(l+1), & j=l+\frac{1}{2}, \\ l, & j=l-\frac{1}{2} .\end{cases}
$$

Hence, $\kappa$ contains the information about both angular momentum quantum numbers $l, j$ that can be obtained from $l=|\kappa|+\frac{1}{2}(\kappa /|\kappa|-1)$ and $j=|\kappa|-1 / 2$. The values of $\kappa$ for the upper and lower spinor spherical harmonics are such that, if the upper spinor has orbital angular momentum $l$, the lower spinor orbital angular momentum should be $\tilde{l}=l-\kappa /|\kappa|$.

To describe the antinucleons, we obtain from Eq. (5) the corresponding conjugate spinor

$$
\psi_{\mathrm{c}}=i \gamma^{2} \psi^{*}=\left(\begin{array}{c}
-F_{n \kappa}(r) i \sigma_{2} \phi_{-\kappa m_{j}}^{*}(\theta, \varphi) \\
i G_{n \kappa}(r) i \sigma_{2} \phi_{\kappa m_{j}}^{*}(\theta, \varphi)
\end{array}\right),
$$

which can still be written as

$$
\psi_{\mathrm{c}}=(-1)^{m_{j}-(\kappa /|\kappa|) / 2+1} i\left(\begin{array}{c}
i F_{n \kappa}(r) \phi_{-\kappa-m_{j}}(\theta, \varphi) \\
-G_{n \kappa}(r) \phi_{\kappa-m_{j}}(\theta, \varphi)
\end{array}\right),
$$

or, after performing the replacements

$$
\begin{aligned}
-\kappa & \rightarrow \bar{\kappa}, \\
F_{n \kappa}(r) & \rightarrow \bar{G}_{\bar{n} \bar{\kappa}}(r), \\
G_{n \kappa}(r) & \rightarrow \bar{F}_{\bar{n} \bar{\kappa}}(r),
\end{aligned}
$$

$$
\psi_{\mathrm{c}}=(-1)^{m_{j}+(\bar{\kappa} /|\bar{\kappa}|) / 2+1} i\left(\begin{array}{r}
i \bar{G}_{\bar{n} \bar{\kappa}}(r) \phi_{\bar{\kappa},-m_{j}}(\theta, \varphi) \\
-\bar{F}_{\bar{n} \bar{\kappa}}(r) \phi_{-\bar{\kappa},-m_{j}}(\theta, \varphi)
\end{array}\right)
$$


We can label the spinor $\psi_{\mathrm{c}}$ using the quantum numbers of its upper component, namely, $\bar{n}, \bar{l}, \bar{j}$. These are to be regarded as the quantum numbers for the charge-conjugated spinor that is the solution of the charge-conjugated Dirac equation (3).

For fermions, the first-order differential radial equations are obtained from Eq. (2) in the usual way, leading to

$$
\begin{aligned}
G_{n \kappa}^{\prime}+\frac{1+\kappa}{r} G_{n \kappa} & =(E+m-\Delta) F_{n \kappa}, \\
F_{n \kappa}^{\prime}+\frac{1-\kappa}{r} F_{n \kappa} & =-(E-m-\Sigma) G_{n \kappa},
\end{aligned}
$$

where $\Delta=V-S$ and $\Sigma=V+S$.

We use the same procedure to obtain the radial differential equations for antifermions from the conjugate Dirac Hamiltonian (3) and spinor (10). One obtains

$$
\begin{gathered}
\bar{G}_{\bar{n} \bar{\kappa}}^{\prime}+\frac{1+\bar{\kappa}}{r} \bar{G}_{\bar{n} \bar{\kappa} \bar{\kappa}}=-\left(E_{\mathrm{c}}-m-\Sigma\right) \bar{F}_{\bar{n} \bar{\kappa}}, \\
\bar{F}_{\bar{n} \bar{\kappa} \bar{\kappa}}^{\prime}+\frac{1-\bar{\kappa}}{r} \bar{F}_{\bar{n} \bar{\kappa}}=\left(E_{\mathrm{c}}+m-\Delta\right) \bar{G}_{\bar{n} \bar{\kappa}} .
\end{gathered}
$$

We observe that these equations for antifermions are very similar to Eqs. (11) for fermions, the main effect of charge conjugation being the transformation $\Delta \rightarrow-\Sigma$ and $\Sigma \rightarrow-\Delta$ as already mentioned in the Introduction. Moreover, there is another important modification: The binding energy for the fermions is given by $\epsilon=E-m$ while the binding energy for antifermions is given by $\epsilon_{\mathrm{c}}=-E_{\mathrm{c}}-m$. In terms of these eigenvalues, Eqs. (11) and (12) become, respectively,

$$
\begin{aligned}
G_{n \kappa}^{\prime}+\frac{1+\kappa}{r} G_{n \kappa} & =(\epsilon+2 m-\Delta) F_{n \kappa}, \\
F_{n \kappa}^{\prime}+\frac{1-\kappa}{r} F_{n \kappa} & =-(\epsilon-\Sigma) G_{n \kappa}
\end{aligned}
$$

and

$$
\begin{aligned}
\bar{G}_{\bar{n} \bar{\kappa}}^{\prime}+\frac{1+\bar{\kappa}}{r} \bar{G}_{\bar{n} \bar{\kappa}} & =\left(\epsilon_{\mathrm{c}}+2 m+\Sigma\right) \bar{F}_{\bar{n} \bar{\kappa}}, \\
\bar{F}_{\bar{n} \bar{\kappa}}^{\prime}+\frac{1-\bar{\kappa}}{r} \bar{F}_{\bar{n} \bar{\kappa}} & =-\left(\epsilon_{\mathrm{c}}+\Delta\right) \bar{G}_{\bar{n} \bar{\kappa} \bar{\kappa}} .
\end{aligned}
$$

It is also instructive to write the second-order differential equations for both the upper and lower components of the fermions and antifermions spinors:

$$
\begin{aligned}
& G_{n \kappa}^{\prime \prime}-\frac{\kappa(\kappa+1)}{r^{2}} G_{n \kappa}+\frac{\Delta^{\prime}}{\epsilon+2 m-\Delta}\left(\frac{d}{d r}+\frac{1+\kappa}{r}\right) G_{n \kappa} \\
& \quad=-(\epsilon-\Sigma)(\epsilon+2 m-\Delta) G_{n \kappa} \\
& F_{n \kappa}^{\prime \prime}-\frac{\kappa(\kappa-1)}{r^{2}} F_{n \kappa}+\frac{\Sigma^{\prime}}{\epsilon-\Sigma}\left(\frac{d}{d r}+\frac{1-\kappa}{r}\right) F_{n \kappa} \\
& \quad=-(\epsilon-\Sigma)(\epsilon+2 m-\Delta) F_{n \kappa}
\end{aligned}
$$

and

$$
\begin{aligned}
& \bar{G}_{\bar{n} \bar{\kappa}}^{\prime \prime}-\frac{\bar{\kappa}(\bar{\kappa}+1)}{r^{2}} \bar{G}_{\bar{n} \bar{\kappa}}-\frac{\Sigma^{\prime}}{\epsilon_{\mathrm{c}}+2 m+\Sigma}\left(\frac{d}{d r}+\frac{1+\bar{\kappa}}{r}\right) \bar{G}_{\bar{n} \bar{\kappa}} \\
& =-\left(\epsilon_{\mathrm{c}}+\Delta\right)\left(\epsilon_{\mathrm{c}}+2 m+\Sigma\right) \bar{G}_{\bar{n} \bar{\kappa}}, \\
& \bar{F}_{\bar{n} \bar{\kappa}}^{\prime \prime}-\frac{\bar{\kappa}(\bar{\kappa}-1)}{r^{2}} \bar{F}_{\bar{n} \bar{\kappa}}-\frac{\Delta^{\prime}}{\epsilon_{\mathrm{c}}+\Delta}\left(\frac{d}{d r}+\frac{1-\bar{\kappa}}{r}\right) \bar{F}_{\bar{n} \bar{\kappa} \bar{\kappa}} \\
& =-\left(\epsilon_{\mathrm{c}}+\Delta\right)\left(\epsilon_{\mathrm{c}}+2 m+\Sigma\right) \bar{F}_{\bar{n} \bar{\kappa} .}
\end{aligned}
$$

From these sets of equations the interchange of roles of $\Delta$ and $\Sigma$ in the equations for the upper and lower radial wave functions for fermions and antifermions is even clearer. For instance, the role of the $\Delta$ potential, which contributes to the effective mass $m^{*}$ of fermions $\left(2 m^{*}=\epsilon+2 m-\Delta\right)$, is played by $-\Sigma$ in the case of antifermions, with a "conjugate" effective mass such that $2 m_{\mathrm{c}}^{*}=\epsilon_{\mathrm{c}}+2 m+\Sigma$. In contrast, the role of the $\Sigma$ potential as the binding potential for fermions is now played by $-\Delta$ for antifermions. We will discuss in the next section the consequences of this role change for the level distribution and the onset of the symmetries of the antinucleon spectrum.

Actually, the sets of equations (13) and (14) are identical provided we make the correspondences $\Sigma \rightarrow-\Delta, \Delta \rightarrow-\Sigma$, and $\epsilon \rightarrow \epsilon_{\mathrm{c}}$ (corresponding to $E \rightarrow-E_{\mathrm{c}}$ ). Therefore, for a given pair $(S, V)$ of potentials the numerical results for a certain positive energy state (the binding energy and wave functions) are the same as for a negative energy state with the same quantum numbers and the pair $(S,-V)$, except, of course, for the total energy $E$ and $E_{\mathrm{c}}$. We recall that the classification of the single-particle levels is given by the quantum numbers of the upper component in both cases; hence a correspondence between the spin and pseudospin partners with positive and negative energies can be made.

The second-order equations (15)-(18) allow us to identify the spin- and pseudospin-orbit terms for both fermions and antifermions [11]. These are

$$
\begin{aligned}
&- \frac{\Delta^{\prime}}{\epsilon+2 m-\Delta} \frac{1+\kappa}{r} G_{n \kappa} \quad \text { (spin), } \\
&-\frac{\Sigma^{\prime}}{\epsilon-\Sigma} \frac{1-\kappa}{r} F_{n \kappa} \quad \text { (pseudospin) }
\end{aligned}
$$

for fermions, and

$$
\begin{gathered}
\frac{\Sigma^{\prime}}{\epsilon_{\mathrm{c}}+2 m+\Sigma} \frac{1+\bar{\kappa}}{r} \bar{G}_{\bar{n} \bar{\kappa}} \quad \text { (spin), } \\
\frac{\Delta^{\prime}}{\epsilon_{\mathrm{c}}+\Delta} \frac{1-\bar{\kappa}}{r} \bar{F}_{\bar{n} \bar{\kappa}} \quad \text { (pseudospin) }
\end{gathered}
$$

for antifermions. These last expressions make clear the role that $\Delta$ and $\Sigma$ potentials play, respectively, in the onset of the spin and pseudospin symmetries for fermions and the reversal of their roles for antifermions. If one divides the second-order equations (15)-(18) by (twice) the effective masses referred to above, one gets Schrödinger-like equations (see Ref. [11]), thereby providing a way of obtaining the contributions of their various terms to the single-particle binding energy $\epsilon$. In particular, one gets for the spin- and pseudospin-orbit contributions for the energy of the level with quantum numbers $n \kappa$ (fermions)

$$
\begin{aligned}
E_{n \kappa}^{\mathrm{SO}} & =-\frac{\int_{0}^{\infty} \frac{\Delta^{\prime}}{\left(\epsilon_{n \kappa}+2 m-\Delta\right)^{2}} \frac{1+\kappa}{r}\left|G_{n \kappa}\right|^{2} r^{2} d r}{\int_{0}^{\infty}\left|G_{n \kappa}\right|^{2} r^{2} d r}, \\
E_{n \kappa}^{\mathrm{PSO}} & =-\frac{\int_{0}^{\infty} \frac{\Sigma^{\prime}}{\left(\epsilon_{n \kappa}-\Sigma\right)\left(\epsilon_{n \kappa}+2 m-\Delta\right)} \frac{1-\kappa}{r}\left|F_{n \kappa}\right|^{2} r^{2} d r}{\int_{0}^{\infty}\left|F_{n \kappa}\right|^{2} r^{2} d r} .
\end{aligned}
$$

In these formulas, the integration is taken in the principal-value sense whenever the denominators of the integrands are zero. 
TABLE I. Single-particle neutron binding energies, in MeV, of two spin and two pseudospin partners for various strengths of the vector potential $V_{0}$. The column for $V_{0}=292.0 \mathrm{MeV}$ corresponds to the best fit to the neutron single-particle energies of ${ }^{208} \mathrm{~Pb}$. The states with zero binding energy are in the continuum.

\begin{tabular}{|c|c|c|c|c|c|}
\hline$V_{0}$ & 0.0 & 100.0 & 200.0 & 292.0 & 300.0 \\
\hline$\Sigma_{0}$ & -358.0 & -258.0 & -158.0 & -66.0 & -58.0 \\
\hline$\Delta_{0}$ & 358.0 & 458.0 & 558.0 & 650.0 & 658.0 \\
\hline$\epsilon\left(2 s_{1 / 2}\right)$ & -322.5962 & -224.9267 & -128.2021 & -41.6087 & -34.3833 \\
\hline$\epsilon\left(1 d_{3 / 2}\right)$ & -326.7516 & -228.7713 & -131.5698 & -44.0704 & -36.7021 \\
\hline$\Delta E$ & 4.1554 & 3.8446 & 3.3677 & 2.4616 & 2.3188 \\
\hline$\epsilon\left(2 g_{9 / 2}\right)$ & -255.2061 & -162.2730 & -72.7440 & -1.3198 & 0.0 \\
\hline$\epsilon\left(1 i_{11 / 2}\right)$ & -266.6147 & -172.3074 & -80.4942 & -3.3984 & 0.0 \\
\hline$\Delta E$ & 11.4086 & 10.0344 & 7.7502 & 2.0786 & \\
\hline$\epsilon\left(1 p_{1 / 2}\right)$ & -338.3051 & -239.6125 & -141.4138 & -52.2731 & -44.6683 \\
\hline$\epsilon\left(1 p_{3 / 2}\right)$ & -338.4124 & -239.7783 & -141.6810 & -52.7714 & -44.2065 \\
\hline$\Delta E$ & 0.1073 & 0.1658 & 0.2672 & 0.4983 & 0.4618 \\
\hline$\epsilon\left(1 h_{9 / 2}\right)$ & -283.3606 & -188.0162 & -94.6341 & -14.1499 & -7.9630 \\
\hline$\epsilon\left(1 h_{11 / 2}\right)$ & -284.4187 & -189.6579 & -97.2570 & -18.5705 & -12.5662 \\
\hline$\Delta E$ & 1.0581 & 1.6417 & 2.6229 & 4.4206 & 4.6031 \\
\hline
\end{tabular}

This is the case for $E^{\mathrm{PSO}}$ because $\epsilon-\Sigma$ is zero for some value of $r$. For antifermions, the formulas are similar:

$$
\begin{aligned}
E_{\bar{n} \bar{\kappa}}^{\mathrm{SO}} & =\frac{\int_{0}^{\infty} \frac{\Sigma^{\prime}}{\left(\epsilon_{\bar{n} \bar{\kappa}}+2 m+\Sigma\right)^{2}} \frac{1+\bar{\kappa}}{r}\left|\bar{G}_{\bar{n} \bar{\kappa} \bar{\kappa}}\right|^{2} r^{2} d r}{\int_{0}^{\infty}\left|\bar{G}_{\bar{n} \bar{\kappa}}\right|^{2} r^{2} d r}, \\
E_{\bar{n} \bar{\kappa}}^{\mathrm{PSO}} & =\frac{\int_{0}^{\infty} \frac{\Delta^{\prime}}{\left(\epsilon_{\bar{n} \bar{\kappa}}+\Delta\right)\left(\epsilon_{\bar{n} \bar{\kappa}}+2 m+\Sigma\right)} \frac{1-\bar{\kappa}}{r}\left|\bar{F}_{\bar{n} \bar{\kappa} \bar{c}}\right|^{2} r^{2} d r}{\int_{0}^{\infty}\left|\bar{F}_{\bar{n} \bar{\kappa}}\right|^{2} r^{2} d r} .
\end{aligned}
$$

We will use these formulas later on to assess the perturbative nature of the spin and pseudospin symmetries for fermions and antifermions.

\section{NUMERICAL RESULTS AND DISCUSSION}

In previous work we used realistic mean-field WoodsSaxon potentials in the Hamiltonian (1) to study the structure of the neutron single-particle spectrum of ${ }^{208} \mathrm{~Pb}[10,11]$. By varying the parameters of the Woods-Saxon potential, namely, its depth, diffusivity, and range, we were able to perform a systematic investigation of the pseudospin energy splittings as a function of those parameters. We concluded that the onset of the pseudospin symmetry in nuclei is dynamical, since it results mainly from cancellations of several terms contributing to the single-particle levels, instead of being a consequence of a small pseudospin-orbit coupling. An equivalent statement is that the pseudospin symmetry in nuclei is nonperturbative, as was pointed out by other authors [13].

In the present work, we follow a similar strategy, using mean-field Woods-Saxon potentials whose diffusivity and range are adjusted to reproduce the neutron single-particle spectrum of ${ }^{208} \mathrm{~Pb}$, but allowing for the vector potential strength $V_{0}$ to vary, and study the resulting spectra for both neutrons and antineutrons. We carry out this program by solving numerically the first-order Dirac equations for fermions and antifermions, that is, Eqs. (13) and (14), and obtaining the radial wave functions and eigenenergies.

TABLE II. The antineutron binding energies $\epsilon_{\mathrm{c}}$ (in $\mathrm{MeV}$ ) and splittings for two spin partners and two pseudospin partners. The parameters are the same as in Table I.

\begin{tabular}{lrrrrr}
\hline \hline$V_{0}$ & 0.0 & 100.0 & 200.0 & 292.0 & \multicolumn{1}{c}{300.0} \\
$\Sigma_{0}$ & -358.0 & -258.0 & -158.0 & -66.0 & -58.0 \\
$\Delta_{0}$ & 358.0 & 458.0 & 558.0 & 650.0 & \multicolumn{1}{c}{658.0} \\
$\epsilon_{\mathrm{c}}\left(\overline{2} \bar{s}_{1 / 2}\right)$ & -322.5962 & -420.7518 & -519.2080 & -609.9713 & -617.8704 \\
$\epsilon_{\mathrm{c}}\left(\overline{1} \bar{d}_{3 / 2}\right)$ & -326.7516 & -425.1387 & -523.7798 & -614.6861 & -622.5967 \\
$\Delta E$ & 4.1554 & 4.3869 & 4.5718 & 4.7148 & 4.7263 \\
$\epsilon_{\mathrm{c}}\left(\overline{2} \bar{g}_{9 / 2}\right)$ & -255.2061 & -349.7786 & -445.3284 & -533.8162 & -541.5317 \\
$\epsilon_{\mathrm{c}}\left(\overline{1} \bar{i}_{11 / 2}\right)$ & -266.6147 & -362.1573 & -458.4515 & -547.4968 & -555.2563 \\
$\Delta E$ & 11.4086 & 12.3787 & 13.1231 & 13.6806 & 13.7246 \\
$\epsilon_{\mathrm{c}}\left(\overline{1} \bar{p}_{1 / 2}\right)$ & -338.3051 & -437.2571 & -536.3716 & -627.6573 & -635.5988 \\
$\epsilon_{\mathrm{c}}\left(\overline{1} \bar{p}_{3 / 2}\right)$ & -338.4124 & -437.3241 & -536.4082 & -627.6713 & -635.6111 \\
$\Delta E$ & 0.1073 & 0.0670 & 0.0366 & 0.0140 & 0.0123 \\
$\epsilon_{\mathrm{c}}\left(\overline{1} \bar{h}_{9 / 2}\right)$ & -283.3606 & -380.3511 & -476.6347 & -566.1890 & -573.9897 \\
$\epsilon_{\mathrm{c}}\left(\overline{1} \bar{h}_{11 / 2}\right)$ & -284.4187 & -379.6942 & -476.9910 & -566.3244 & -574.1078 \\
$\Delta E$ & 1.0581 & 0.6569 & 0.3563 & 0.1354 & 0.1181 \\
\hline \hline
\end{tabular}


We used the following parameters, adjusted to ${ }^{208} \mathrm{~Pb}: S_{0}=$ $-358.0 \mathrm{MeV}$ for the depth of $S$, and $a_{v}=a_{s}=0.6 \mathrm{fm}$ and $R_{v}=R_{s}=7.0 \mathrm{fm}$ for, respectively, the diffusivity and range of both $V$ and $S$ potentials [10,11]. We vary $V_{0}$, covering a broad range of values for the binding potential. The parameter $V_{0}=292.0 \mathrm{MeV}$ corresponds to the best fit to the neutron spectrum of ${ }^{208} \mathrm{~Pb}$.

In Table I the binding energies $\epsilon$ and the energy splittings $\Delta E$ for two pseudospin and two spin partners of nucleon single-particle states are shown, for a vector potential strength varying from $V_{0}=0$ to $V_{0}=300.0 \mathrm{MeV}$. As the magnitude of $V_{0}$ increases, the magnitude (absolute value) of $\Sigma_{0}=S_{0}+$ $V_{0}$ decreases and the energy splittings between pseudospin partners become smaller. Concomitantly, the magnitude of $\Delta_{0}=V_{0}-S_{0}$ increases and the energies of the spin partners move away from each other. These results are in agreement with those of previous work [11], and they occur because, for nucleon systems, the $\Sigma(\Delta)$ potential drives the pseudospin(spin)-orbit interaction. Moreover, one sees that, since $\Sigma$ is the binding potential, for $\left|\Sigma_{0}\right|<66.0 \mathrm{MeV}$, the potential becomes too shallow and the higher energy states become unbound.

In Table II we present the same quantities for antineutrons, namely, the binding energies $\epsilon_{\mathrm{c}}$ and the energy splittings for the same two spin and pseudospin partners, using the same values of $V_{0}$ as in Table I. This time, as the magnitude of $\Sigma_{0}$ decreases (and thus $\Delta_{0}$ increases) the energies of the spin partners become quasidegenerate, whereas the energies of the pseudospin partners move away from each other as was already remarked by Zhou et al. [5]. This can be readily explained by the reversal of the role played by the $\Sigma$ and $\Delta$ potentials in the spin and pseudospin symmetries, as explained before. In contrast to the neutrons, for antineutrons the energies of conjugate spin partners become almost degenerate because of the smallness of the $\Sigma$ potential as compared to the $\Delta$ potential, which drives the spin splitting for neutrons.

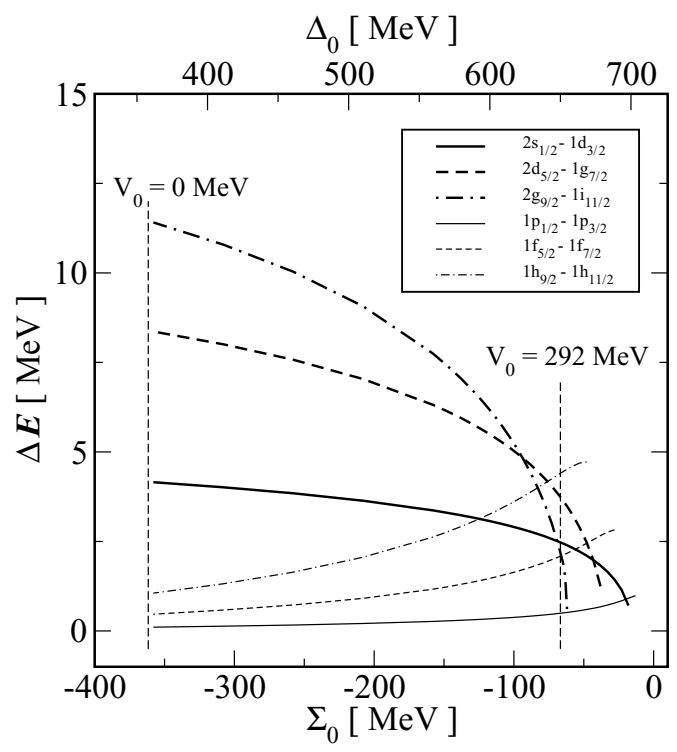

FIG. 1. Splittings for spin and pseudospin partners for neutrons The dashed line with $V_{0}=292 \mathrm{MeV}$ represents the physical region.

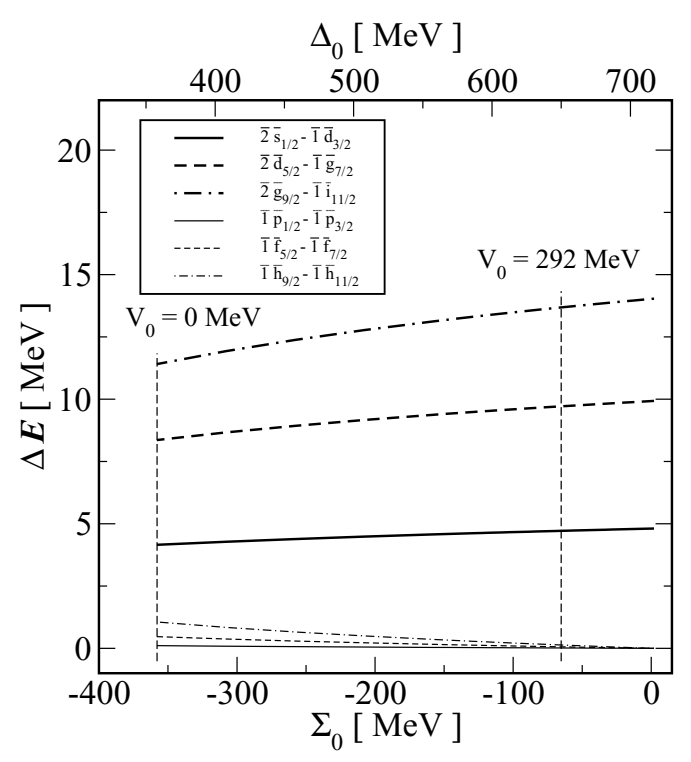

FIG. 2. Splittings for spin and pseudospin partners for antineutrons. The dashed line with $V_{0}=292 \mathrm{MeV}$ represents the physical region.

Because of the correspondence that can be established between the radial equations for particles and for antiparticles mentioned at the end of Sec. II, it is interesting to note that if we computed the energies and splittings for $V_{0}<0$, in the case of the neutrons, for a spin or pseudospin partner, we would get the same values as for the corresponding antineutron spin or pseudospin partner with the positive symmetric value $\left|V_{0}\right|$, and vice versa (the results for antineutrons with $V_{0}<0$ are equal to the results for fermions with the symmetric value $\left.\left|V_{0}\right|\right)$. In other words, for the corresponding single-particle states of positive

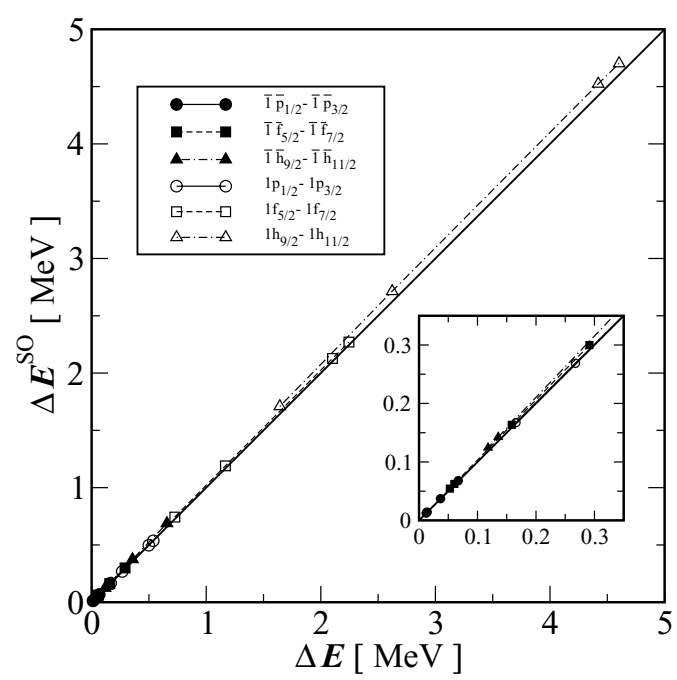

FIG. 3. Spin-orbit term splittings $\Delta E^{\mathrm{SO}}$, that is, the difference in the energies of the terms (19) (neutrons) and (21) (antineutrons), for three spin partners plotted against their respective energy splittings $\Delta E$. The thicker solid line represents the values for which $\Delta E^{\mathrm{SO}}=$ $\Delta E$. The calculation is done for the four positive values of $V_{0}$ in Tables I and II. 


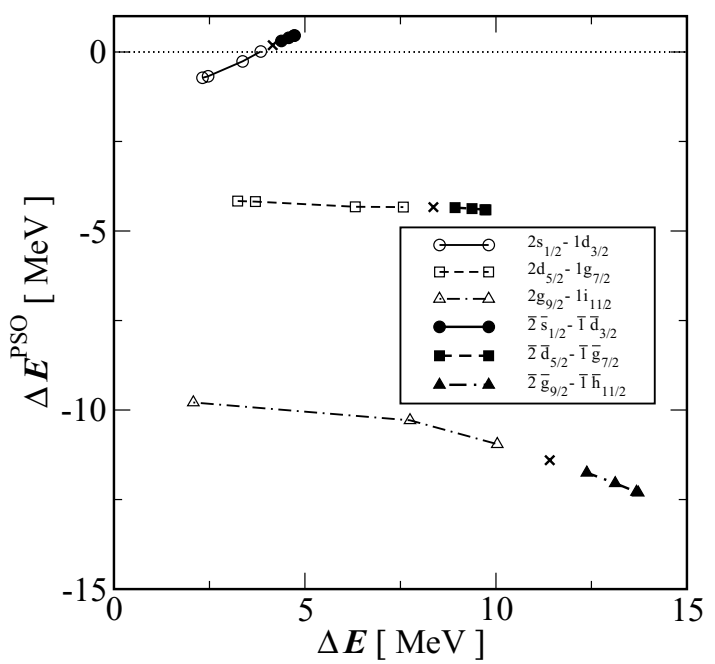

FIG. 4. Pseudospin-orbit term splittings $\Delta E^{\mathrm{PSO}}$, that is, the difference in the energies of the terms (20) (neutrons) and (22) (antineutrons), for three spin partners plotted against their respective energy splittings $\Delta E$. The calculation is done for the four positive values of $V_{0}$ in Tables I and II whenever bound states exist. The points labeled by " $\times$ " mark the $V_{0}=0$ point for each pair of levels.

and negative energies, the behavior of neutrons for $V_{0}>0$ is the same mutatis mutandis as that for antineutrons with $V_{0}<0$, and vice versa. Actually, this correspondence can already be seen from Tables I and II, since for $V_{0}=0$ the single-particle states of neutrons and antineutrons with the same quantum numbers should have the same binding energies; and indeed this is what comes out from the calculations.

We now examine in some more detail both symmetries and their dependence on the central potentials $\Sigma$ and $\Delta$ for neutrons and antineutrons. In Fig. 1 we show the splittings $\Delta E$ for three spin and pseudospin partners as a function of $\Sigma_{0}$, the binding potential depth. The vertical dashed line for $V_{0}=292 \mathrm{MeV}$ stands for the parameters that best reproduce the experimental single-particle energies of ${ }^{208} \mathrm{~Pb}$. For large absolute values of $\left|\Sigma_{0}\right|$ the spin symmetry is better realized because the strength of the $\Delta$ potential, $\Delta_{0}$, becomes smaller and thus the spin-orbit interaction gets weaker. As $\left|\Sigma_{0}\right|$ decreases, the spin-orbit interaction gets more and more important and the deviation from the exact symmetry gets bigger. In the same figure we also represent the splittings for the pseudospin partners. The pseudospin symmetry never becomes an exact one, although the splitting for the partners chosen stay close to zero in the physical region. But this is definitely not the case for large $\Sigma_{0}$. From the same figure one also concludes that the quality of both spin and pseudospin symmetries, as measured by the splittings, is similar for ${ }^{208} \mathrm{~Pb}$. However, there is clearly a different behavior of the spin and pseudospin splittings, respectively, when $\Delta_{0}$ decreases and when $\left|\Sigma_{0}\right|$ decreases.

A similar analysis for antineutrons can be made by looking at Fig. 2, where the splittings for three pseudospin and spin partners with the same quantum numbers as in Fig. 1 are shown again as a function of $\Sigma_{0}$. One sees that for antineutrons the spin symmetry for small $\Sigma_{0}$ (physical region) is very good. On the contrary, pseudospin symmetry is broken significantly. This, of course, was to be expected because of the roles that the $\Sigma$ and $\Delta$ potentials now play in the onset of spin and pseudospin symmetries. From this figure and Fig. 1 one can also see that, as remarked before, for $V_{0}=0$ the antinucleon levels have the same splittings as the corresponding neutron levels. This is because in this case $\Sigma=-\Delta$, and therefore the charge-conjugation operation $\Delta \rightarrow-\Sigma \quad \Sigma \rightarrow-\Delta$ does not change the potentials. The total energy spectrum of the neutrons is then exactly symmetric with the total energy spectrum of the neutrons $\left(E_{\mathrm{c}}=-E\right)$.

In Figs. 3 and 4 are shown the splittings (i.e., the differences) of the (pseudo)spin-orbit [(P)SO] terms Eqs. (19) and (20) (neutrons) and Eqs. (21) and (22) (antineutrons) for three (pseudo)spin partners. The calculations were made for the four positive values of $V_{0}$ shown in Tables I and II. From these figures one can clearly see the different natures of spin and pseudospin in nuclei, for both neutrons and antineutrons. There is a correlation between the values of the spin-orbit coupling and the energy splittings for spin partners, the ratio $\Delta E^{\mathrm{PS}} / \Delta E$ being very close to 1 for antineutrons. This an unmistakable sign of the perturbative nature of spin symmetry in nuclei, for both neutrons and antineutrons.

The situation for the pseudospin partners is completely different. There is no correlation between the pseudospin term splittings and the energy splittings, even for small values of $\Delta E$. We see that even the sign is different in most cases. Therefore, in spite of the fact mentioned earlier that there is a connection between the strength of the $\Sigma$ $(\Delta)$ potential and neutron (antineutron) pseudospin energy splittings, there is not a relation between the corresponding pseudospin-orbit terms and the energy splittings, that is, the onset of pseudospin symmetry. Thus we can conclude that the
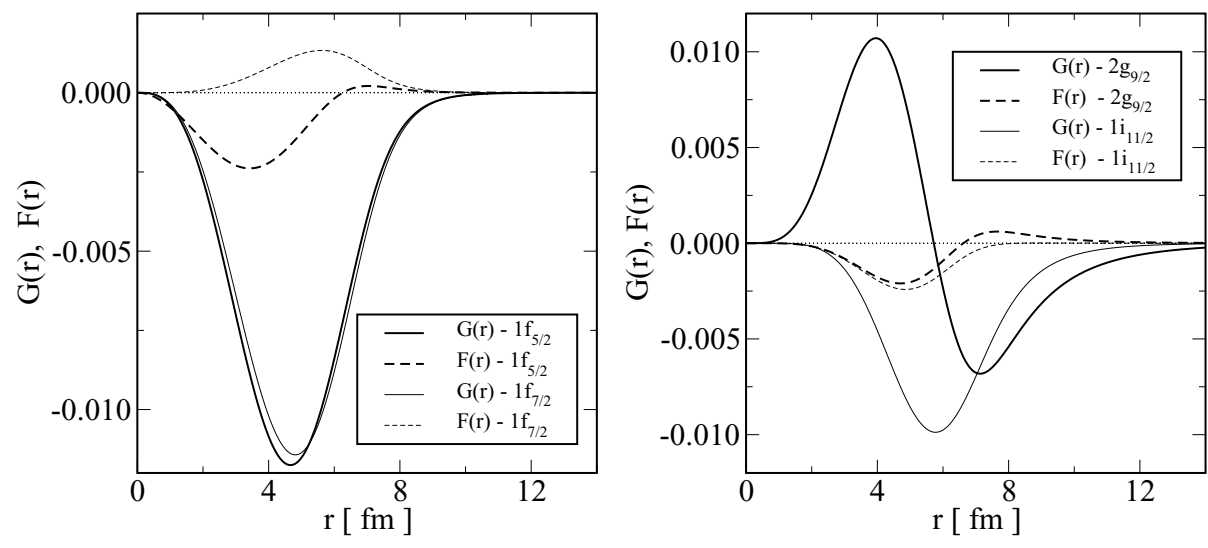

FIG. 5. Neutron radial wave functions $G$ and $F$ of the spin pair $1 f_{5 / 2^{-}}$ $1 f_{7 / 2}$ and the pseudospin pair $2 g_{7 / 2-}$ $1 i_{11 / 2}$ for the physical parameters in scaled units. 

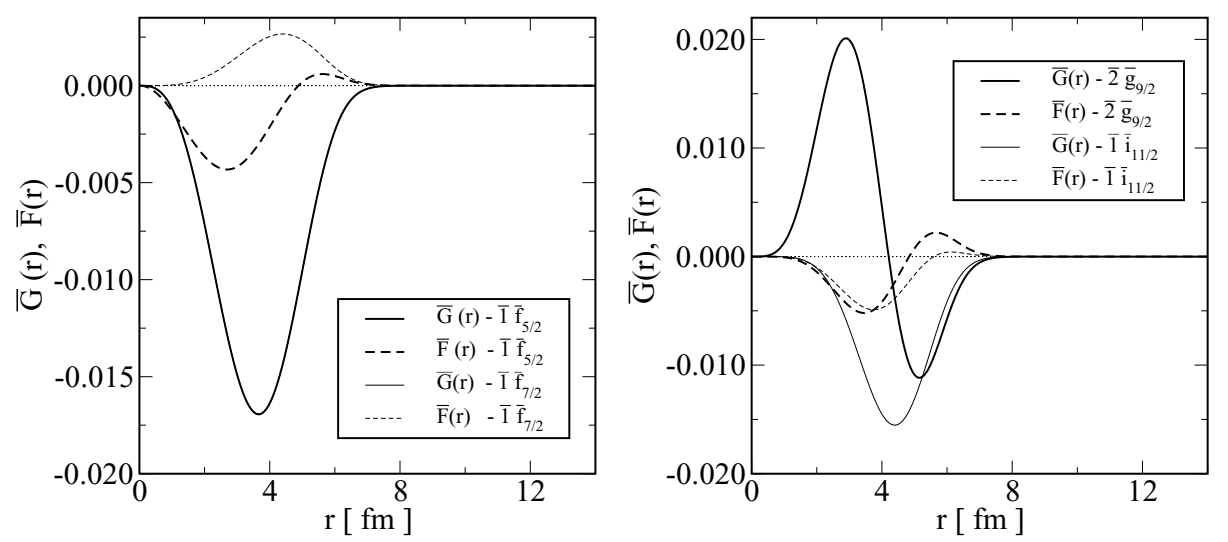

FIG. 6. Antineutron radial wave functions $G$ and $F$ of the spin pair $\overline{1} \bar{f}_{5 / 2}-\overline{1} \bar{f}_{7 / 2}$ and the pseudospin pair $\overline{2} \bar{g}_{7 / 2}-\overline{1} \bar{i}_{11 / 2}$ for the physical parameters in scaled units. pseudospin symmetry in nuclei is not perturbative, for either neutrons or antineutrons.

Finally, we present the radial functions $G$ and $F$ of one spin doublet and one pseudospin doublet. We chose the pairs $\left[1 f_{5 / 2}\right.$ $\left.1 f_{7 / 2}\right]$ and $\left[2 g_{7 / 2}-1 i_{11 / 2}\right]$ because they have about the same energy splittings for neutron levels for the physical parameters.

One sees from Fig. 5 that the spin and pseudospin pair degeneracy for neutrons is related to the similarity of the $G$ and $F$ wave functions, respectively. This can be understood by just remarking that the second-order equations for these functions, having the same quantum numbers for each pair, are basically the same when the eigenvalues are very similar, which implies, for normalized wave functions, that the corresponding solutions should also very similar.

This can also be seen from Fig. 6 for the spin doublet, which is so highly degenerate that the two radial functions $\bar{G}$ can hardly be distinguished. Comparing the two figures, one can also see that the amplitude of the upper radial functions $\bar{G}$ is bigger for the antineutron case. This is basically due to the increased kinetic energy of the antineutrons, which one can roughly estimate by the difference between the depth of the potential well and the binding energy.

This analysis of the radial functions can assess only the amount of degeneracy of corresponding doublets and not the perturbative nature of the respective symmetries. There are some possibilies for performing such an analysis using the radial functions, as was done by Marcos et al. [14], but they are rather indirect. The similarity of the pseudospin partners' radial functions $F$ as a signature for pseudospin symmetry was already shown by Ginocchio and Madland in [15].

\section{CONCLUSIONS}

In this paper, we systematically examined the spin and pseudospin symmetries in nuclei for realistic Woods-Saxon potentials that fit the neutron single-particle spectrum of ${ }^{208} \mathrm{~Pb}$. This analysis covers both the nucleon and the antinucleon spectra, obtained by charge conjugation of the Dirac equation for mean-field scalar and vector potentials. By solving the Dirac equation for neutrons and antineutrons for several values of the depth of the vector potential, we were able to perform a systematic analysis of spin and pseudospin symmetries by computing the energy splittings of the corresponding doublets, as well as computing the spin- and pseudospin-orbit contributions to those splittings. From that analysis, one concludes that spin symmetry, besides being almost exact for antinucleons, is perturbative and thus can be realized exactly. On the other hand, pseudospin symmetry, for both nucleons and antinucleons, is found to be not perturbative. This is probably related to the fact that the potential that drives this symmetry is, in both cases, also the binding potential, and thus one cannot have bound states in the conditions of exact pseudospin symmetry for the type of potentials studied in this paper, which go asymptotically to zero.

The main difference between the spectra of single-particle levels of neutrons and antineutrons is the much increased depth of the potential (by about ten times) and the kinetic energies. In this sense, the antinucleon bound states are more relativistic than the nucleon states. However, the main features of the onset of spin and pseudospin symmetry are the same, the differences being basically quantitative.

\section{ACKNOWLEDGMENTS}

We acknowledge financial support from $\mathrm{CNPq}$, the Capes-FCT Project No. 183/07, and also the Projects No. PTDC/FIS/64707/2006 and No. CERN/FP/83505/2008 from FCT.
[1] J. N. Ginocchio, Phys. Rep. 414, 165 (2005).

[2] R. Lisboa, M. Malheiro, A. S. de Castro, P. Alberto, and M. Fiolhais, Phys. Rev. C 69, 024319 (2004).

[3] T. Bürvenich, I. N. Mishustin, L. M. Satarov, J. A. Maruhn, H. Stöcker, and W. Greiner, Phys. Lett. B 542, 261 (2002).

[4] A. Martin et al., Nucl. Phys. A 487, 563 (1988).
[5] S.-G. Zhou, J. Meng, and P. Ring, Phys. Rev. Lett. 91, 262501 (2003).

[6] A. S. de Castro, P. Alberto, R. Lisboa, and M. Malheiro, Phys. Rev. C 73, 054309 (2006).

[7] J. N. Ginocchio, Phys. Rev. C 69, 034318 (2004).

[8] J. N. Ginocchio, Phys. Rep. 315, 231 (1999).

[9] C.-Y. Song, J.-M. Yao, and J. Meng, Chin. Phys. Lett. 26, 122102 (2009). 
[10] P. Alberto, M. Fiolhais, M. Malheiro, A. Delfino, and M. Chiapparini, Phys. Rev. Lett. 86, 5015 (2001).

[11] P. Alberto, M. Fiolhais, M. Malheiro, A. Delfino, and M. Chiapparini, Phys. Rev. C 65, 034307 (2002).

[12] C. Itzykson and J. B. Zuber, Quantum Field Theory (McGrawHill, New York, 1980).
[13] S. Marcos, L. N. Savushkin, M. López-Quelle, and P. Ring, Phys. Rev. C 62, 054309 (2000).

[14] S. Marcos, V. N. Fomenko, M. Lopez-Quelle, R. Niembro, and L. N. Savushkin, Eur. Phys. J. A 26, 253 (2005).

[15] J. N. Ginocchio and D. G. Madland, Phys. Rev. C 57, 1167 (1998). 